\title{
Anotaciones sobre el Adenocarcinoma de Cervix
}

\author{
HOSPITAL UNIVERSITARIO SAN VICENTE DE PAUL - MEDELLIN \\ XVII CONGRESO COLOMBIANO DE OBSTETRICIA Y GINECOLOGIA \\ Pereira - Diciembre 1o. al 5 de 1987 \\ Dres.: Gildardo Gallego Noreña* y Jaime Uribe Duque**
}

\section{INTRODUCCION}

Del Carcinoma Epidermoide de Cervix se ha investigado bastante en sus aspectos etiológicos, diagnóstico precoz, vías de diseminación, tipo de tratamiento, mortalidad, morbilidad, seguimiento, otros. Pero aún hay muchas incógnitas por resolver y serán motivo de futuras investigaciones.

Una variedad histológica de los carcinomas cervicales son los de tipo glandular, específicamente el adenocarcinoma. Sabemos poco sobre esta variedad histológica y poco se ha investigado hoy. Una revisión completa de la literatura colombiana desde 1970 , demuestra la ausencia completa de este tema.

Motivado personalmente en este aspecto, considero importante hacer este estudio descriptivo, retrospectivo con el fin de hacer un diagnóstico del estado actual del adenocarcinoma de cervix y que sirva como base para un trabajo prospectivo de investigación.

\section{OBJETIVO GENERAL}

1. Analizar nuestra experiencia sobre el adenocarcinoma de cervix, y tratar de hacer un diagnóstico del estado actual de esta neoplasia.

Profesor Asociado 750 - Departamento de Obstetricia y Ginecología Facultad de Medicina - Universidad de Antioquia.

Profesor Ad-honorem - Departamento de Obstetricia y Ginecología. Facultad de Medicina - Universidad de Antioquia.
2. Con base en el análisis, elaborar un protocolo prospectivo de investigación.

\section{OBJETIVOS ESPECIFICOS}

1. Determinar y analizar variables relacionadas con:

1.1. Grupos de Edad

1.2. Paridad

1.3. Clasificación Clínica por Estadios

1.4. Síntoma Principal de Consulta

1.5. Método Diagnóstico Empleado

1.6. Antecedente de Ingesta de Anovulatorios

1.7. Patología Asociada

1.8. Tipo de Tratamiento

1.9. Tiempo de seguimiento

2. Determinar número de casos por año.

\section{MATERIAL Y METODOS}

Con el fin de conocer nuestra experiencia en el Hospital Universitario San Vicente de Paúl, sobre el adenocarcinoma cervical, teniendo en cuenta que en la literatura colombiana no hay ningún informe al respecto, consideramos importante realizar esta revisión, que nos permita un diagnóstico del estado actual del adenocarcinoma cervical y planear ajustes necesarios para mejorar el manejo y seguimiento de esta entidad.

En el Grupo de Oncología Ginecológica del Departamento de Obstetricia y Ginecología de la 
Facultad de Medicina de la Universidad de Antioquia, figuran registradas en el computador $80 \mathrm{pa}-$ cientes con diagnóstico confirmado por anatomía patológica de adenocarcinoma cervical desde el 1 o. de enero de 1980 hasta el 31 de julio de 1987. En este mismo período aparecen registradas $2.624 \mathrm{pa}-$ cientes con diagnóstico de carcinoma de cervix. La Tabla 1, nos da una idea de la relación del adenocarcinoma con el epidermoide.

\section{TABLA 1}

\section{GANCER DE CUELLO UTERINO GRUPO ONCOLOGIA GINECOLOGICA 1980 - 1987}

\begin{tabular}{|l|r|c|}
\hline TIPO CARCINOMA & No CASOS & $\%$ \\
\hline CANCER EPIDERMOIDE & 2.541 & 96 \\
\hline CANCER INDIFERENCIADO & 3 & 1.1 \\
\hline ADENOCARCINOMA & 80 & 3 \\
\hline TOTAL & 2.624 & 100 \\
\hline
\end{tabular}

Estos datos dan una relación del 3\% para el adenocarcinoma del cervix, con referencia al número total de casos.

\subsection{Grupos de edad}

En la gráfica 1, se aprecia que los grupos etáreos de mayor frecuencia con adenocarcinoma de cervix fueron los comprendidos entre 30 y 60 años.

Las pacientes más jóvenes fueron entre 24 y 25 años y la de más edad fue de 75 años con un X de edad de 46 años.

No se puede concluir que el adenocarcinoma de cervix se presente en pacientes más jóvenes. Por el contrario, en el Hospital Universitario San Vicente de Paúl, se tiene registrado carcinoma epidermoide en pacientes tan jóvenes como de 20 años.

En el estudio de Taimimi (16) la paciente más joven con adenocarcinoma fue de 15 años y la más anciana de 83 años con un promedio de $43.6 \%$.

\subsection{Paridad}

Se puede apreciar que la mayoría de las pacientes fueron multíparas; agrupadas de uno a cua-

\section{GRAFICA 1}

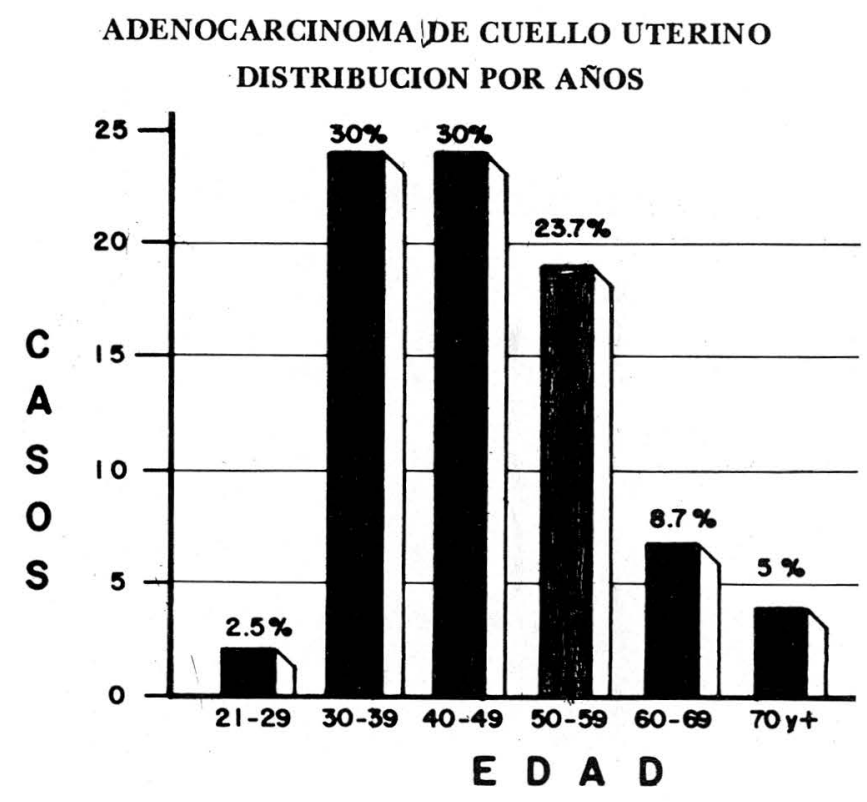

GRAFICA 2

HOSPITAL UNIVERSITARIO SAN VICENTE DE PAUL $1980-1987$

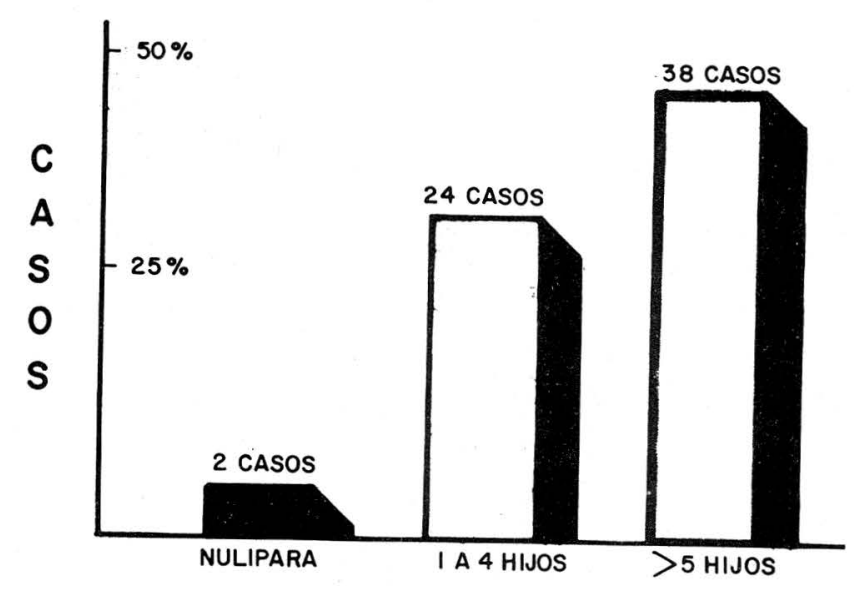

tro hijos $(30 \%)$ y más de cinco hijos 38 pacientes $(47.5 \%)$. Sólo hubo dos casos en pacientes nulíparas. En 16 pacientes no aparece consignado la paridad debido a que como este no es un estudio prospectivo, carece de formulario especial.

Taimimi y Col. (16) registra 22\% (13 pacientes) de nulíparas que presentaron adenocarcinoma de cervix. Una hipótesis de trabajo futuro sería pro- 
bar que el adenocarcinoma de cervix es más frecuente en multíparas.

Dos pacientes estaban en embarazo en el momento del diagnóstico y fueron sometidas a histerectomía radical.

\subsection{Clasificación clínica por estadios}

Estadio clínico: adenocarcinoma de cervix

Tradicionalmente se han ordenado los carcinomas de acuerdo con la clasificación internacional (FIGO). Aparecen tres casos (3.7\%) sin catalogar, posiblemente fueron remitidas por médicos particulares o ya estaban operadas.

Observamos un gran porcentaje de pacientes para estadios I B 25 pacientes (31.2\%), II B 17 pacientes $(21.2 \%)$ y III B 28 pacientes $(35 \%)$.

En cambio en las variedades "A" aparecen clasificados sólo tres casos como se aprecia en la tabla. Sabemos que el adenocarcinoma tiende a ser más de tipo endofítico y por esta razón se disemina más frecuentemente a parametrios que a vagina.

\subsection{Síntoma principal: adenocarcinoma de cervix}

Específicamente se investigó en las historias lo referente a síntoma principal como causa de consulta. Esta información es pobre. Algunas pacientes consultaron únicamente para toma de citología rutinaria. Los síntomas principales fueron hemorragia vaginal, leucorreas, dolor en hipogastrio.

A continuación aparecen en la tabla 2.

En cincuenta pacientes no se encontró ningún tipo de síntoma. Se sabe que el cáncer cérvico-uterino es silencioso desde el punto de vista clínico. Sería conveniente investigar sistemáticamente las manifestaciones clínicas más comunes para poder obtener datos concretos.

\subsection{Métodos diagnósticos}

La ayuda diagnóstica más utilizada fue la biopsia de lesiones cervicales. Este dato fue consignado en 52 historias $(65 \%)$. En algunas pacientes se rea-
TABLA 2

CLASIFICACION CLINICA - $1980-1987$ 80 CASOS

\begin{tabular}{|c|c|c|}
\hline \multicolumn{2}{|c|}{ ESTADIO } & $\%$ \\
\hline \multirow{2}{*}{1} & & 5.00 \\
\hline \multirow{2}{*}{ II } & A & 1.20 \\
\cline { 2 - 3 } & B & 31.20 \\
\hline \multirow{2}{*}{ III } & B & 1.20 \\
\cline { 2 - 3 } & B & 21.20 \\
\hline \multirow{2}{*}{ IV } & & 35.00 \\
\hline \multirow{2}{*}{ Indeterminado } & & 1.20 \\
\hline TO TAL & & 3.70 \\
\hline
\end{tabular}

TABLA 3

SINTOMAS

\begin{tabular}{|l|c|}
\hline \multicolumn{1}{|c|}{ SINTOMA } & No Pacientes \\
\hline HEMORRAGIA VAGINAL & 16 \\
\hline DOLOR HIPOGASTRICO & 7 \\
\hline LEUCORREA & 7 \\
\hline
\end{tabular}

lizaron otros métodos diagnósticos como citología, colposcopia, cono (ver gráfica 3).

Podemos apreciar que la biopsia de cuello fue un método diagnóstico muy frecuente, posiblemente debido a que la lesión era macroscópicamente visible en un gran número de casos. I B, II B, III B (70 casos) en contraposición, el curetaje endocervical se realizó sólo en un caso, cuando en realidad es 
ideal este método para lesiones que están en el canal endocervical o cuando no se observa totalmente la unión escamocolumnar a la visión colposcópica. El curetaje endocervical mejoraría el diagnóstico hasta descubrir las lesiones in situ.

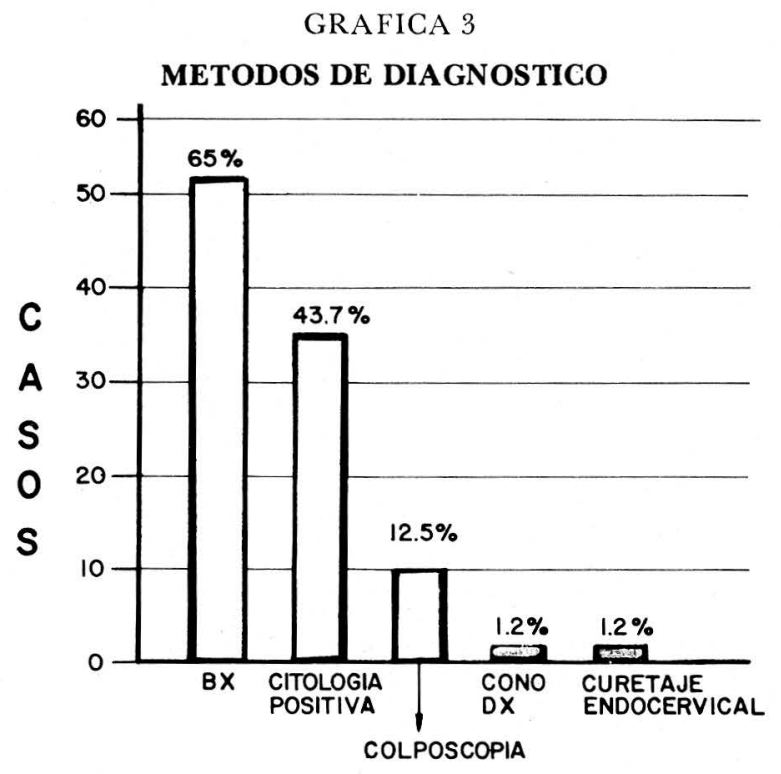

\subsection{Anovulatorios}

Varios estudios han tratado de relacionar el antecedente de ingesta de anovulatorios y adenocarcinoma cervical. El uso prolongado de éstos puede ser un factor desencadenante $(9,16,15,4)$.

Sólo encontramos el antecedente de planificar con anovulatorios en cinco historias. Un estudio prospectivo debe analizar tipo de anovulatorios, concentración estrogénica, tiempo de exposición, otros y relacionarlos con adenocarcinoma cervical.

Se postula que el adenocarcinoma cervical tenga algún factor desencadenante diferente al de la variedad escamosa, probablemente algún factor hormonal común o relacionados para el carcinoma endometrial, pero hasta el momento no se ha podido demostrar.

\subsection{Patología asociada}

En la Tabla 4, aparece registrada la patología asociada. La mayoría de las historias son pobres en estos datos, pues no hay formato específico para consignar variables relacionadas.
TABLA 4

PATOLOGIA ASOCIADA

\begin{tabular}{|l|c|}
\hline \multicolumn{1}{|c|}{ PATOLO I A } & CASOS \\
\hline $\begin{array}{l}\text { CANCER EPIDERMOIDE INVASOR } \\
\text { DE CERVIX Y ADENOCARCINOMA }\end{array}$ & 1 \\
\hline HIPERTENSION ARTERIAL & 2 \\
\hline ADENOMIOSIS & 1 \\
\hline ENDOMETRIOSIS & 1 \\
\hline
\end{tabular}

\subsection{Tipo de tratamiento}

El tratamiento fue quirúrgico en algunos casos, radioterapia en otros o cambiando en un determinado número de casos.

Se realizó histerectomía radical (Wertheim) en 16 casos $(20 \%)$ para las clasificadas como estadio I B. No todas las pacientes con este estadio son tributarias de cirugías porque algunas tienen contraindicaciones quirúrgicas. De las pacientes sometidas a Wertheim, el informe de ganglios fue negativo en quince casos y positivo en un caso a quien se trató complementariamente con radioterapia.

Se realizó anexohisterectomía en seis pacientes $(7.5 \%)$ e histerectomía simple en cinco $(6.25 \%)$ posiblemente para las pacientes con adenocarcinoma in situ.

El tratamiento estuvo basado en la clasificación clínica. Lo ideal como en el carcinoma epidermoide, sería descubrirlo en las formas in situ y así obtendríamos mejor pronóstico.

Se trataron con Cobalto y Radium en forma combinada (externa e intracasitariamente) 51 pacientes del I B en adelante.

Universalmente se sabe que el adenocarcinoma de cervix es más radiorresistente. Entonces el tratamiento quirúrgico sería de elección. Cuatro pacientes se trataron con radioterapia externa únicamente. Una con terapia intracavitaria, tres sin tratamiento posiblemente por su estadio muy avanzado o se trataron particularmente. 
GRAFICA 4

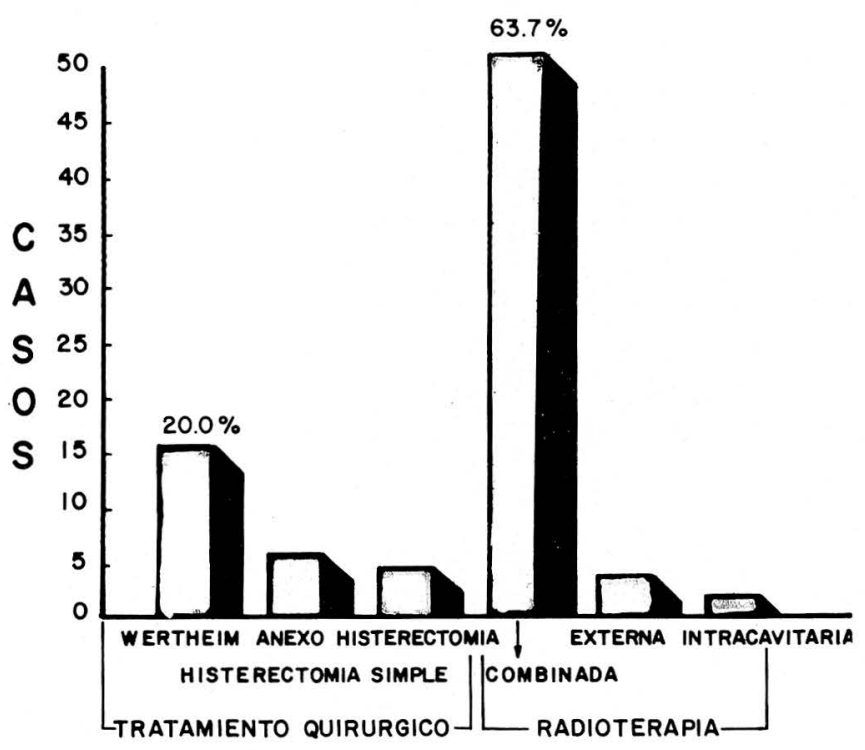

Anatomía patológica del espécimen quirúrgico:

En las pacientes sometidas a cirugía se pudo constatar el espécimen quirúrgico en 23 de ellas.

Como puede apreciarse en la Tabla 5, el adenocarcinoma invasor fue el encontrado en la mayoría de los casos, posiblemente los relacionados con el tratamiento quirúrgico tipo Wertheim.

TABLA 5

PATOLOGIA DEL ESPECIMEN QUIRURGICO

\begin{tabular}{|l|c|c|}
\hline TIPO DE CANCER & CONO & HISTERECTOMIA \\
\hline CANCER EPIDERMOIDE IN SITU & & 1 \\
\hline CANCER EPIDERMOIDE INVASOR & & 1 \\
\hline ADENOCARCINOMA IN SITU & 1 & 3 \\
\hline ADENOCARCINOMA INVASOR & & 17 \\
\hline ADENOESCAMOSO & & 1 \\
\hline
\end{tabular}

\subsection{Tiempo de seguimiento}

En la Tabla 6, apreciamos un 50\% de pacientes seguida menos de doce meses, en parte explicable porque en los dos últimos años hubo un número mayor de pacientes con diagnóstico de adenocarcinoma de cervix.
TABLA 6

MESES DE SEGUIMIENTO

\begin{tabular}{|c|c|c|}
\hline MESES DE SEG & N & $\%$ \\
\hline MENOS DE I2 MESES & 40 & 50 \\
\hline 12 Y 24 MESES & 15 & 18.7 \\
\hline 25 Y 36 MESES & 2 & 2.5 \\
\hline 36 Y 48 MESES & 10 & 1.2 \\
\hline 48 Y 60 MESES & 13 & 16.2 \\
\hline TO TAL & 80 & 100 \\
\hline
\end{tabular}

El número de casos registrados de adenocarcinoma por año va en aumento a medida que transcurren los años. Pero este dato puede ser casual o servir de base hipotética para un estudio prospectivo. La Gráfica 5 es muy ilustrativa de la tendencia al aumento.

\section{GRAFICA 5}

HOSPITAL UNIVERSITARIO SAN VIGENTE DE PAUL $1980-1987$

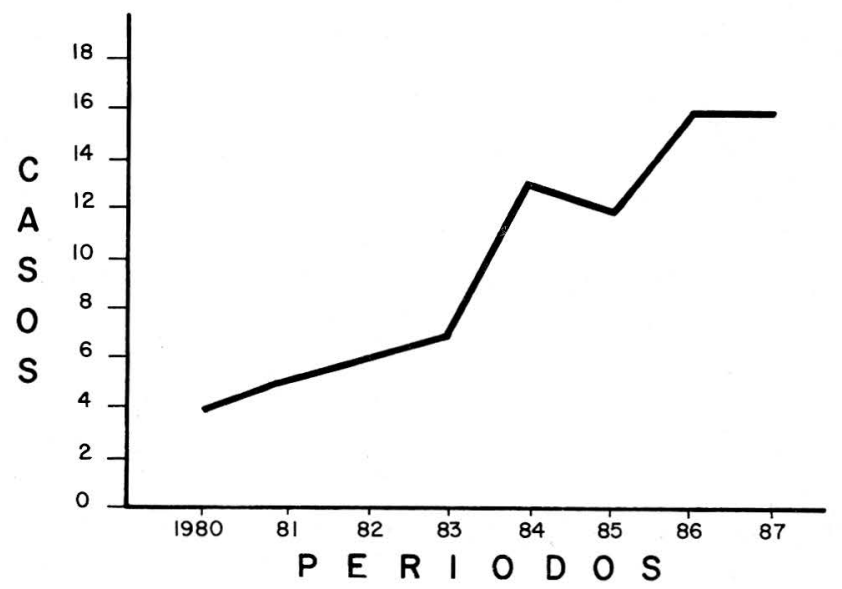

COMENTARIOS Y DISCUSION

\section{Etiología}

Poco se sabe acerca del origen del adenocarcinoma de cuello. Las asociaciones de actividad sexual 
y factores virales atribuidos para el carcinoma de células escamosas, parece que no intervienen en la génesis del adenocarcinoma (1). Sin embargo, no hay un estudio que lo niegue o apruebe rotundamente.

\section{Factores hormonales}

¿Obedece al adenocarcinoma a una respuesta hormonal $(6,15)$ ?

Algunos investigadores han sostenido que el adenocarcinoma cervical obedece a factores hormonales y que se encuentra más asociado a pacientes embarazadas. Gallup and Abel (6) en una serie de 33 pacientes con adenocarcinoma de cérvix, sólo una estaba embarazada y en la presente revisión del Hospital Universitario San Vicente de Paúl, de 80 adenocarcinomas, sólo dos estaban embarazadas en el momento del diagnóstico.

¿Los anovulatorios orales desencadenarían el adenocarcinoma de cervix?

Se ha demostrado que los anticonceptivos orales por períodos cortos de tiempos son inocuos y se ha probado el efecto protector para algunas entidades tales como las lesiones benignas de mama (9). En cambio el uso de ellos por largos períodos de tiempo, puede constituirse en factor etiológico desencadenante y será necesario comprobar esta posible relación causa-efecto.

En el Hospital Universitario San Vicente de Paúl, el dato de ingesta de anovulatorios sólo estaba consignado en cinco pacientes pero no se sabía el tipo de anovulatorios y período de uso.

Taimimi (16) informa historia positiva de ingesta de anovulatorios en 16 a 32 pacientes cuyas edades estaban por debajo de los 40 años. Dallenbach y Hellweg (4) informan 28 adenocárcinomas cervicales en un período de diez años y encontraron que el $23 \%$ de estas pacientes habían tomado anticonceptivos orales con promedio de uno a diez años. En este estudio, nueve pacientes con adenocarcinoma in situ habían tomado anticonceptivos con potente gestágeno. cervical?
Se ha tratado de asimilar características del carcinoma de endometrio al adenocarcinoma de cervix (13). Así se ha dicho que el adenocarcinoma de cervix es más frecuente en nulíparas. Para Milsom y Friberg en 67 pacientes con adenocarcinoma cervical fue estadísticamente más significativo la frecuencia en nulíparas. La experiencia en el Grupo de Oncología del Departamento de Obstetricia y Ginecología de la Facultad de Medicina de la Universidad de Antioquia, es opuesta. De las 80 pacientes analizadas, ninguna era nulípara y un $42 \%$ tenían de uno a cuatro hijos y el $44 \%$ tenían más de cinco hijos. (¿Qué factor influye?).

Una hipótesis de trabajo sería comprobar cómo influye la paridad, la obesidad, la hipertensión y la diabetes, en la génesis del adenocarcinoma cervical.

\section{Pronóstico}

El adenocarcinoma de cervix tiene peor pronóstico que el tumor escamoso celular. Varios estudios lo confirman $(13,15,16)$, principalmente porque se detecta en una forma más tardía, debido a su localización anatómica (canal endocervical) y clínicamente más asintomático.

Tiene más tendencia a la invasión ganglionar, por este motivo las recidivas locales son frecuentes.

IV. Criterios para la identificación del adenocarcinoma primario del cervix

Kaminski Paul informa varios criterios para identificar el adenocarcinoma de cervix; éstos son:

A. Identificar en el espécimen, zona de transición de glándulas endocervicales.

B. Encontrar endometrio normal en muestras de curetaje o espécimen de histerectomía.

C. Encontrar adenocarcinoma en el muñón cervical cinco o más años después de la histerectomía subtotal.

D. Tumor macroscópico localizado en el cervix y ausencia de tumor macroscópico en la cavidad endometrial. 
V. Bases histológicas del adenocarcinoma cervical

En el diagnóstico histológico de lesiones glandulares del cervix hay dos variables de importancia pronóstica: la profundidad de la invasión, o sea, si se trata de una lesión in situ, superficial o profundamente invasora y la diferenciación celular, o sea, hasta donde las células recuerda la célula de la cual se origina la neoplasia.

Con relación al origen ontogénico, se consideran tres grupos: los de células endocervicales; los de origen en células de reserva y los de restos mesonéfricos.

Como principio general se acepta como lesión in situ, aquella en la que se observa proliferación del epitelio glandular con cualquier grado de alteración celular. Si la lesión es puramente glandular, se denomina adenocarcinoma in situ. Si penetra hasta $5 \mathrm{~mm}$ el estroma, se denomina cáncer glandular temprano o microinvasor. Necesariamente debe excluir radiación previa y el estudio circunferencial del cuello es el requerimiento para el diagnóstico definitivo.

Son tumores bien diferenciados los que se forman de glándulas que tienen muy poca desviación con el patrón glandular normal del endocervix. Muestran hipercromasia nuclear y poca mitosis.

Los moderadamente diferenciados tienen glándulas con ramificación compleja. Los mal diferenciados tienen una estructura de glándulas o seudotúbulos sólidos y difícilmente recuerdan el origen glandular.

La gran mayoría tiene origen en glándulas del endocervix y en ellos el grado de diferenciación tiene significado pronóstico. No sucede lo mismo con los de origen en células de reserva que tiene peor pronóstico que los de glándula endocervical aunque sean diferenciados.

Los de remanentes mesonéfricos son siempre mal diferenciados, crecen profundamente en el cuello y tienen peor pronóstico $(3,5,14,17)$.
Necesariamente suponemos que el patólogo esté bien entrenado en cuanto al reconocimiento de estas lesiones y esto nos ayudará a enfocar en forma adecuada el tratamiento a seguir.

El tratamiento efectuado se basó principalmente en la clasificación clínica. Sería óptimo descubrir el adenocarcinoma en el estado in situ. Al igual que el epidermoide en el mismo estadio aseguraría un buen pronóstico.

Como dato sobresaliente se aprecia la tendencia creciente del número de adenocarcinoma de cervix a medida que transcurren los años y esta sería una hipótesis futura.

\section{CONCLUSIONES}

De la presente revisión concluimos:

1. El adenocarcinoma de cervix es una entidad que ocupa el $3 \%$ de todos los carcinomas de cuello.

2. Se encontró principalmente en el grupo de la tercera, cuarta y quinta década de la vida.

3. Fue más frecuente en multíparas.

4. Ideal descubrirlo en las formas in situ.

5. No se puede sacar ninguna conclusión en cuanto a síntomas y relación de anticonceptivos con el adenocarcinoma de cuello.

6. Se debe mejorar el diagnóstico en cuanto a colposcopia y curetaje endocervical.

7. Hay una tendencia al aumento en el número de adenocarcinoma de cervix a medida que pasan los años; ilustrado por la presente revisión y por la revisión de la literatura mundial $(6,12,13,15,16)$. 


\section{BIBLIOGRAFIA}

1. ANDERSON MALCOLM. "Etiología del Cáncer de Cuello Uterino" Temas Actuales, VIII, 1976.

2. AD COCK, L. "Carcinoma of the cervix, figo stage I B: Treatment failures”. Gynec. Onc. 18 (2) 218-25, 1984.

3. BURGHARDT E. "Early histologyc diagnosis of cervical cancer" Saunders, 1973.

4. DALLENBACH H. "On the origin and histological structure of adenocarcinoma of the endocervix in women under $\mathbf{5 0}$ years of age". Pathol Res. Pract.: 179 (1) 38-50, 1984.

5. SAINICHI T. "Early stage adenocarcinoma of the uterine cervix cancer” 56. 167-172, 1985.

6. GALLUP D. "Invasive adenocarcinoma of the uterine cervix" Obst. Gynec. Vol. 49 No. 5, May. 1977.

7. GALLUP D. "Poor prognosis in patients with adenosquamans A II carcinoma of the cervix" Obst. Gynec. Vol. 65 (3) March 1985.

8. HILL E. "Radical surgery in the management of clear A II adenocarcinoma of the cervix and vagina in young women". Am. J. Obst. Gyn. May. 15, 1981.
9. HUGGINS G. "Neoplasias y anticonceptivos hormonales". Clin. Obst. y Gin. Vol. 3. 1981.

10. KYORSTAD K. "Stage I B adenocarcinoma of the cervix. Metastatic potencial and pattern of dissemination". Am. J. Obst. Gyn. Vol. 130 (3) Oct. 1o, 1984.

11. KAMINSKI P. "Clear cell adenocarcinoma of the cervix unrelated to diethestilbestrol exposure". Obst. Gynec. Vol. 62 No. 6, December, 1983.

12. MENCZER J. "Adenocarcinoma of the uterine cervix in jewish women”. Cáncer 41: 2464-67, 1978.

13. MILSOM I F. "Primary adenocarcinoma of the cervix uterine" Cáncer 52 (5) 942-7, 1983.

14. MARTZLOFF “Ca. cervix uterine” Bull John Hopkins Hospital 34-141, 1980.

15. SHINGLETON HUGN "Adenocarcinoma of the cervix". Am. J. Obst. Gynec. Vol. 139 No. 7, April 1981.

16. TAIMIMI H. "Adenocarcinoma of the uterine cervix" Gynec. Oncol. 13: 335-344, 1982.

17. YARBRO H. "Seminars in Oncology" Vol. IX (3) September, 1982. 